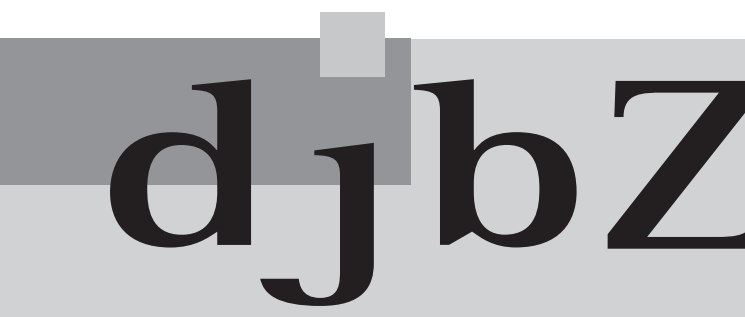

Zeitschrift des

Deutschen

Juristinnenbundes

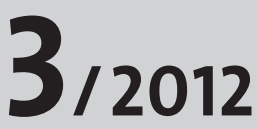

15. Jahrgang, Seite $97-146$

Herausgeber: Deutscher Juristinnenbund e. V.

Präsidium: Ramona Pisal, Vorsitzende Richterin am Oberlandesgericht, Brandenburg a.d.H. (Präsidentin); Margarete Hofmann, Direktorin in der EU-Kommission Brüssel; Eva Schübel, Bundesanwältin beim BGH, Karlsruhe (Vizepräsidentinnen);

Dagmar Brinkmann, Referatsleiterin, Wiesbaden (Schatzmeisterin); Jutta Wagner, Rechtsanwältin und Notarin, Fachanwältin für Familienrecht, Berlin (Past President); Schriftleitung: Anke Gimbal, Rechtsassessorin, Geschäftsführerin Deutscher Juristinnenbund, Berlin.

\title{
Gesetz zur Durchsetzung der Entgeltgleichheit im Parlament
}

\section{Prof. Dr. Heide Pfarr \\ Kassel}

Die SPD-Bundestagsfraktion hat am 14. Juni 2012 einen Gesetzentwurf zur Durchsetzung der Entgeltgleichheit in den Bundestag eingebracht. ${ }^{1}$ Der Abstand zwischen den Entgelten von Frauen und Männern von immerhin 23 Prozent beruht auf verschiedenen, in der Regel gleichfalls diskriminierenden Gründen wie horizontaler und vertikaler Segregation und familienbedingten Erwerbsunterbrechungen und -reduzierungen, aber eben in erheblichem Maße auch auf einem Verstoß gegen das Gebot der Entgeltgleichheit. ${ }^{2}$ Das Gesetz soll diese rechtswidrige Praxis endlich beseitigen. Die SPD hat sich damit einer überaus komplexen Regelungsmaterie angenommen. Denn Entgeltzahlungen haben regelmäßig mehrere Quellen, für die verschiedene Akteur/inn/e/n zuständig sind: Tarifverträge, Vereinbarungen zwischen Betriebsrat und Arbeitgeber/in, einseitig von Arbeitgeber/in gesetzte betriebliche Regelungen und schließlich einzelvertragliche Vereinbarungen, die typischerweise bei strukturellem Ungleichgewicht der Vertragspartner/innen abgeschlossen wurden. Hinzu kommt, dass Entgeltdiskriminierung heute zumeist nicht direkt, sondern mittelbar erfolgt und nicht leicht zu erkennen und nachzuweisen ist. Das bisher geltende Recht unterwirft zwar alle Entgeltfestlegungen einschließlich der Tarifverträge dem Entgeltgleichheitsgebot, es stellt aber kein effektives Verfahren zur Überprüfung und Korrektur zur Verfügung; darüber hinaus fehlt es in der Regel an Transparenz. Die Durchsetzung des Entgeltgleichheitsgebots wird vielmehr an die sozial Schwächsten, die Diskriminierten, überantwortet bzw. denen, die gleichzeitig die Interessenvertretung der bevorzugten Beschäftigtengruppe sind: die Betriebsräte, die ihre Rechte kaum jemals einsetzen. ${ }^{3}$

\section{Bedingungen für ein Entgeltgleichheitsgesetz}

Ein Gesetz zur Durchsetzung der Entgeltgleichheit muss die beiden größten Schwachstellen des geltenden Rechts aufgreifen. Es muss Einblick in die Entgeltfestlegung gewähren und Akteur/inn/e/n mit Rechten ausstatten, die nicht den Abhängigkeiten und Einschränkungen unterliegen, die zurzeit die Durchsetzung des Entgeltgleichheitsgebots verhindern. Beides ist überaus kompliziert, denn Informationspflichten kollidieren mit Datenschutz und staatlicher gestaltender Zugriff auf betriebliche Entgeltsysteme ist weder machbar noch erstrebenswert. Tarifverträge genießen als Ausdruck der grundrechtlich verbürgten Koalitionsfreiheit weitgehenden Schutz vor Eingriffen. Im Hinblick auf die grundsätzlich mögliche Einschaltung von Gerichten ist Zurückhaltung geboten. Denn würden Arbeitsgerichte mit der Ersetzung diskriminierender Entgeltsysteme durch rechtmäßige Alternativen betraut, würde damit eine Regelungsfrage wie eine Rechtsfrage behandelt. Praktisch kommt aber fast nie nur eine einzige alternative Regelung in Betracht; es gibt stets Spielräume für geschlechtergerechte Entgeltsysteme, die zu bewerten und zu nutzen nicht Aufgabe der Arbeitsgerichte ist. Gleichzeitig ist und bleibt aber die Arbeitsgerichtsbarkeit die Instanz, die für die Durchsetzung gesetzlicher Handlungspflichten unverzichtbar ist.

Das Gesetz der SPD stellt sich diesen Herausforderungen und versucht, durch ein gestaffeltes System von Verpflichtungen, Beratungen und Kontrollen Entgeltgleichheit in der Pra-

\footnotetext{
1 BT-Drs. $17 / 9781$ v. 23.5.2012.

2 Projektgruppe GiB, Geschlechterungleichheiten im Betrieb. Arbeit, Entlohnung und Gleichstellung in der Privatwirtschaft, Berlin 2010, S. 282; Winter, Regine, „Diskriminierungsfreie(re) Entgeltgestaltung - leider immer noch ein Prinzip ohne Praxis“, in HohmannDennhardt, Christine/Körner, Marita/Zimmer, Sabine, Geschlechtergerechtigkeit. Festschrift für Heide Pfarr, Berlin 2010, S. 320 ff.

3 Vgl. ausführlich GiB, a.a.O., S. 487 ff.
} 
xis durchzusetzen. Dabei belässt es die Entgeltfestlegung bei denen, die nach unserem Rechtssystem dafür verantwortlich sind. Das sind die Geschäftsführungen und die Betriebsräte, soweit es Letztere gibt. Das sind auch die Gewerkschaften und Arbeitgeberverbände, die entsprechende Tarifverträge abschließen. Diesen Akteur/inn/en bleibt die aktive Verantwortung für das Entgelt und damit für die Entgeltgleichheit und die notwendigen Korrekturen. Vereinfacht gesagt verpflichtet das Gesetz die Verantwortlichen zur Analyse ihrer Entgeltsysteme unter sachverständiger Begleitung, zur Offenlegung der Ergebnisse und, für den Fall, dass Entgeltdiskriminierung festgestellt wurde, diese in einem festgelegten Verfahren unter Beteiligung der betrieblichen Interessenvertretung zu beseitigen. Das klingt zunächst einfach, verlangt aber durchstrukturierte Verfahrensregeln und kommt ohne Kontrolle nicht aus. So ist ein Gesetz formuliert worden, das auf den ersten Blick kompliziert erscheinen mag, dessen Regelungstiefe aber wegen der Kompliziertheit der Rechtsmaterie unverzichtbar ist, um effektiv einwirken zu können.

\section{Herstellung von Transparenz der betrieblichen Entgeltpraxis}

Das Gesetz verpflichtet die/den Arbeitgeber/in, in bestimmten Abständen die betriebliche Entgeltpraxis einer Prüfung zu unterziehen. Der Betriebsrat und, soweit vorhanden, die/ der Gleichstellungsbeauftragte sind zu beteiligen. Das Prüfungsverfahren wird gesetzlich vorgeschrieben und auf dem Verordnungswege konkretisiert. Praxisnahe Methoden, um Entgeltdifferenz zu ermitteln, sind inzwischen entwickelt worden ${ }^{4}$ und liefern ausreichende Informationen. Ohne diesen Fortschritt in der geschlechtergerechten Arbeitsbewertung und der EDV-gestützten Analyse von Entgeltsystemen wäre ein effektives Gesetz zur Durchsetzung der Entgeltgleichheit nicht möglich. Grundlage für die Überprüfung auf Entgeltdiskriminierung sind betriebliche Daten über Entgeltsysteme und Entgeltpraxis. Die Verantwortlichen für Entgeltsysteme haben in der Regel allerdings kein Interesse daran, Informationen für eine Überprüfung selbst zu erheben oder zur Verfügung zu stellen, wenn damit Entgeltungleichheit festgestellt würde oder dies auch nur zu befürchten ist. So machen von dem kostenlosen Angebot des Bundesfamilienministeriums, freiwillig die Entgeltgleichheit mittels der computergestützten Methode Logib-D ${ }^{5}$ zu überprüfen, gerade einmal 200 Unternehmen (von ca. 400.000) Gebrauch. Voraussetzung jedes Tätigwerdens sowohl der innerbetrieblichen wie der außerbetrieblichen Akteur/inn/e/n ist aber die Transparenz der betrieblichen Entgeltsysteme und der Entgeltpraxis. Das Gesetz verlangt doch eigentlich nur das, was rechtstreue Betriebe von sich aus tun müssten, um Entgeltungleichheit zu vermeiden bzw. zu beseitigen. Denn wenn sie nicht bewusst und gezielt diskriminieren wollen, gibt es auch für sie selbst keinen anderen Weg, um versteckte, mittelbare Diskriminierung aufzudecken.

\section{Prüfung der betrieblichen Entgeltpraxis}

Das Gesetz sieht als ersten Schritt eine kursorische Prüfung der betrieblichen Entgeltpraxis vor, die anhand der erhobenen betrieblichen Daten mit einem Computerprogramm durchgeführt werden soll. Die Arbeitgeber/innen können im Einvernehmen mit der betrieblichen Interessenvertretung wählen, ob diese kursorische Prüfung durch die Antidiskriminierungsstelle des Bundes (ADS) oder durch eine sachverständige Person im Betrieb durchgeführt wird. Das gesetzliche Angebot einer Prüfung durch die ADS ist der Überlegung geschuldet, dass kleinere und Mittelbetriebe die Kosten für den Einsatz der sachverständigen Person sparen können sollen. Prüft die ADS, die dafür qualifiziertes Personal benötigt, müssen dieser die notwendigen Daten zur Verfügung gestellt werden. Die kursorische Prüfung kann durch EDV-Einsatz so gestaltet werden, dass sie nicht mehr als ca. 15 Minuten dauert. Der Personaleinsatz bleibt somit durchaus überschaubar - dennoch ist vorauszusehen, dass gegen diesen Gesetzentwurf nicht nur der übliche Einwand übermäßiger Bürokratie, sondern auch zu hoher Kosten erhoben werden wird. Ergeben sich aus der kursorische Prüfung des Entgeltberichts Verdachtsmomente auf Entgeltdiskriminierung, verlangt das Gesetz eine detaillierte Prüfung, deren Inhalt durch Verordnung geregelt werden würde. Warum nicht von Anfang an eine detaillierte Prüfung? Im Gegensatz zu der kursorischen Prüfung ist diese eingehender und verlangt recht großen Aufwand. Dieser soll vermieden werden, wenn er nicht unbedingt notwendig erscheint. Insbesondere dann, wenn nach Ablauf der im Gesetz verankerten Frist erneut eine Prüfung der betrieblichen Entgeltpraxis stattfinden muss, reicht in der Regel eine kursorische Prüfung, insbesondere dann, wenn zuvor ein Verfahren zur Herstellung der Entgeltgleichheit durchgeführt worden war.

Die detaillierte Prüfung ist in einem durch die ADS zertifizierten Verfahren durch eine sachverständige Person vorzunehmen, die die/der Arbeitgeber/in (im Einvernehmen mit dem Betriebsrat) aus der Liste der zertifizierten sachverständigen Personen bei der ADS auszuwählen und zu betrauen hat. Der Prüfungsbericht ist mit anonymisierten Daten im Betrieb zu veröffentlichen, um so schon eine interne Kontrolle zu ermöglichen. Er ist gleichermaßen an die ADS zu übermitteln.

\section{Einbeziehung sachverständiger Personen}

Das Gesetz überträgt damit einer sachverständigen Person für Entgeltgleichheit eine wesentliche Rolle. Das ist auch sachgerecht. Denn die Einbeziehung von sachkundigen Personen für eine diskriminierungsfreie Entgeltgestaltung ist schon angesichts der Schwierigkeit der Materie und der verbreiteten Unkenntnisse über diskriminierende Faktoren in Entgeltsystemen unabdingbar. Die sachverständige Person muss von der ADS zertifiziert sein. Die Anforderungen folgen dem Beispiel des Einsatzes von Wirtschaftsprüfer/inne/n in Unternehmen. Wie diese nimmt sie quasi ein öffentliches Amt wahr, soll also nicht nur beraten, sondern auch den für Entgeltfestsetzungen Verantwortlichen auf die Finger schauen. Mehrere Regelungen im

4 Vgl. dazu Tondorf, Karin/Jochmann-Döll, Andrea, „,Von der Entgeltdifferenz zur Entgeltgleichheit der Geschlechter?“, in: WSI-Mitteilungen 2011 (3), S. 115 ff.

5 Die allerdings untauglich ist, siehe Tondorf, Karin, djbZ 2009, S. $130 \mathrm{ff}$. 
Gesetz sollen die Unabhängigkeit und Einwirkungsmöglichkeiten der sachverständigen Person gewährleisten.

\section{Einwirkungsmöglichkeiten außerbetrieblicher Akteur/inn/e/n}

Das Gesetz reagiert ferner auf die Tatsache, dass die Verantwortlichen für die Entgeltsysteme ihrer Rechtspflicht zur Einhaltung des Entgeltgleichheitsgebots nicht aus eigenem Antrieb nachzukommen pflegen, so dass es einer außerbetrieblichen Kontrolle und Durchsetzung bedarf. Einwirkungsmöglichkeiten auf die Verantwortlichen, die nicht entsprechend tätig werden, begründet das Gesetz für Antidiskriminierungsverbände (Definition wie im AGG) und ADS, daneben auch verstärkt für Tarifvertragsparteien und Betriebsräte. Diese können mithilfe der Arbeitsgerichtsbarkeit bzw. Bußgeldern erzwingen, dass die betrieblichen Überprüfungs- und Korrekturprozesse in Gang gesetzt werden.

Die notwendigen Informationen für eine außerbetriebliche Einwirkung bilden ein Problem, das sicher nicht nur datenschutzrechtliche Einwände begründen könnte. Entgeltregelungen in Tarifverträgen sind im Prinzip öffentlich, ihre betrieblichen Umsetzungen hingegen schon nicht mehr. Ein Zugriff außerbetrieblicher Akteur/inn/e/n auf Informationen über betriebliche Entgeltsysteme und betriebliche Entgeltpraxis, die nicht allgemein zugänglich sind, ist unabdingbar, kann jedoch wegen des Datenschutzes nur durch Einschaltung einer staatlichen Stelle gelöst werden, die sensible Daten aus den Betrieben vor dem Einblick von außen solange schützt, wie für Interventionen wegen Rechtsverstößen keine Veranlassung besteht. Ist hingegen Entgeltungleichheit festgestellt, ändert sich die Interessenabwägung: Ein Geheimhaltungsinteresse des Unternehmens kann dann keinen Vorrang mehr haben. Auch ein Gesetz, das auf Selbstkontrolle und Selbstkorrektur setzt und vor allem zivilgesellschaftliche Akteur/inn/e/n für die Überprüfung und Durchsetzung heranziehen will, kommt daher nicht ganz ohne eine staatliche Instanz aus.

Um eine außerbetriebliche Kontrolle und Einwirkung zu ermöglichen, erstellt die ADS Listen derjenigen Betriebe, die keine Prüfungsberichte eingereicht haben oder bei denen Entgeltdiskriminierung festgestellt wurde; sie führt auch eine Liste derjenigen Betriebe, bei denen keine Entgeltdiskriminierung festgestellt oder diese beseitigt worden ist. Diese Listen sind einsehbar. Auf der Basis dieser Information können zivilgesellschaftliche Akteur/inn/e/n tätig werden. Das wären also Gewerkschaften, zuständige Arbeitgeberverbände, Betriebsräte, Beschäftigte des Betriebes und - informiert durch die Listen der ADS - schließlich als weitere neue Akteur/inn/e/n Antidiskriminierungsverbände.

Das Gesetz legt schließlich Ablauf und Beteiligte des Verfahrens zur Korrektur diskriminierender Entgeltsysteme fest. Dabei ist das Verfahren unterschiedlich, je nachdem, ob es im Betrieb einen Betriebsrat gibt oder nicht. Denn das Gesetz muss darauf bedacht sein, den Betriebsrat und seine Mitbestimmungsrechte nicht etwa zu ersetzen oder auszuhebeln. Insbesondere wenn Antidiskriminierungsverbände tätig werden, darf es nicht zu einer verdrängenden Konkurrenz kom- men. Betriebsräte sind so zu unterstützen, dass sie Erfolg versprechend an der Aufgabe, Entgeltgleichheit herzustellen, arbeiten können.

\section{Verfahren zur Korrektur diskriminierender Entgeltpraxis}

Das Gesetz folgt dem arbeitsrechtlichen Pfad; es sieht die Feststellung der Entgeltdiskriminierung als Rechtsfrage, die Schaffung eines diskriminierungsfreien betrieblichen Entgeltsystems aber als Regelungsfrage. Ist Entgeltdiskriminierung festgestellt, ist eine Einigungsstelle für Entgeltgleichheit zu bilden, ganz ähnlich der Einigungsstelle des Betriebsverfassungsgesetzes, also mit der gleichen Anzahl von Beisitzerinnen und Beisitzern von Arbeitgeber- und Betriebsratsseite. Die Gleichstellungsbeauftragte und die zuständigen Tarifvertragsparteien sind zu beteiligen. Darüber hinaus muss das Verfahren durch die sachverständige Person begleitet werden, die die Prüfung durchgeführt hat. Den Vorsitz übernimmt eine sachkundige unparteiliche Person, auf die sich die Betriebsparteien einigen müssen, gleichfalls geregelt wie im Betriebsverfassungsgesetz.

Die Einigungsstelle für Entgeltgleichheit hat die Aufgabe, die Ansprüche auf Nachzahlung vorenthaltenen Entgelts festzustellen, diskriminierende Entgeltpraxis zu beseitigen sowie das System diskriminierungsfrei zu gestalten; dabei kann sie alle Regelungen der Entgeltsysteme und Entgeltpraxis verabschieden, die auf betrieblicher Ebene getroffen werden können. Hat die Entgeltdiskriminierung ihre Grundlage in einer tariflichen Regelung, fehlt auf der betrieblichen Ebene die Befugnis zur Regelung. Die Einigungsstelle kann dann nur feststellen, dass die diskriminierenden tariflichen Regelungen nicht anwendbar sind und deshalb den Diskriminierten die gleichen Ansprüche zustehen wie den begünstigten Beschäftigten. Das gilt aber nur so lange, bis die Tarifvertragsparteien diskriminierungsfreie Regelungen vereinbart haben.

\section{Pay Equity Plan}

Die Rechtsprechung hat bisher in den (wenigen) Entgeltgleichheitsverfahren, die Individualansprüche betrafen, den Benachteiligten das höhere Entgelt der Begünstigten zugesprochen. Für Ansprüche in der Vergangenheit bedeutete das immer die Anpassung der Entgelte nach oben. Dies erscheint einleuchtend und nur gerecht. Die sofortige Angleichung der Entgelte nach oben, um Gleichbehandlung herzustellen, kann aber für zukünftige Entgeltzahlungen zum Problem werden. Dies ist zum einen dann der Fall, wenn es dadurch in einem personalkostenintensiven Betrieb zu einem so erheblichen Kostenschub kommt, dass die wirtschaftliche Grundlage des Unternehmens möglicherweise unterminiert wird. Das wäre ein Dilemma. Denn einerseits kann an einem Zusammenbruch des Unternehmens auch den bislang Diskriminierten nicht liegen, andererseits würde eine solche Folge auch belegen, in welch großem Ausmaß der bisherige wirtschaftliche Erfolg des Unternehmens auf der Diskriminierung von Beschäftigten beruht. Die sofortige Anpassung diskriminierender Entgelte nach oben kann ferner dann problematisch sein, wenn es sich, wie häufig, um komplexe, in sich austarierte Entgeltsysteme 
handelt, in die mittelbar diskriminierende Elemente eingebettet sind. Eine punktuelle Neubewertung verschöbe das bisherige Gleichgewicht - was ja im Sinne der Beseitungung struktureller Diskriminierung an sich gewollt ist. Dies sollte jedoch systematisch und behutsam angegangen werden, damit es nicht zu neuen Ungereimtheiten und möglicherweise zu weiteren und anderen Diskriminierungen führt. Komplexe Entgeltsysteme können daher oft nicht lediglich in einzelnen Regelungen korrigiert, sondern müssen insgesamt abgelöst und durch ein neues, geschlechtergerechtes Entgeltsystem ersetzt werden. Dies ist besonders kompliziert, wenn Diskriminierungsfreiheit mit abgesicherten Besitzständen in Einklang gebracht werden muss. Das geht nur mithilfe eines Entgelt-Anpassungsplans ( „Pay Equity Plan“). ${ }^{6}$

Das Gesetz lässt derartige Anpassungspläne und damit auch Stufenlösungen zu, also eine schrittweise Anpassung der Entgelte nach oben und nach unten. Das bedeutet allerdings, dass die Diskriminierung noch für einen gewissen überschaubaren Zeitraum fortgesetzt wird. Eine solche Stufenplanregelung ist mit der Rechtsprechung des EuGH zu vereinbaren, da der EuGH in einem neueren Urteil $^{7}$ eine zeitlich begrenzte Fortführung der Diskriminierung im Rahmen einer befristeten Übergangslösung als zulässig erachtet hat. Der EuGH hatte zwar über eine tarifliche Regelung zu entscheiden. Seine Aussagen sind aber auf betriebliche Regelungen dann zu übertragen, wenn auch hier die vom EuGH verlangten Voraussetzungen gegeben sind. Danach muss der Stufenplan erforderlich sein, er muss in einer ausgeglichene Machtstruktur zustandegekommen sein und das Verfahren muss mit dem Ziel des Ausgleiches unterschiedlicher Interessen ablaufen. Die im Gesetz vorgesehene Einigungsstelle für Entgeltgleichheit, ihre Besetzung und die Beteiligung der Tarifvertragsparteien gewährleisten dies. Das bedeutet gleichzeitig, dass Stufenpläne nur dann zulässig sind, wenn die Entscheidungen durch die Beteiligung einer betrieblichen Interessenvertretung kontrolliert und mitgestaltet werden können; sie werden im Gesetz deshalb lediglich in solchen Betrieben zugelassen, in denen ein Betriebs- oder Personalrat besteht und mitwirkt.

Übrigens: selbst Stufenlösungen in moderaten Anpassungsplänen werden Betriebsräte und Gewerkschaften durchaus vor Probleme stellen. Denn für die Beseitigung von bisheriger Entgeltdiskriminierung ist es praktisch unvermeidlich, zukünftige Entgelterhöhungen auf jeden Fall ausschließlich oder in ganz überwiegendem Maße den bisher Benachteiligten zugute kommen zu lassen. Das macht die Gewerkschaft für die bisher Begünstigten nicht gerade attraktiv. Die Erfahrung lehrt auch, dass selbst eine Besitzstandsregelung für Männer, die allerdings bei Entgelterhöhungen abgeschmolzen werden soll, das Betriebsklima schwer belastet kann. Denn es fehlt regelmäßig an dem Bewusstsein bei Männern, zu Unrecht profitiert zu haben. Schon heute greifen nicht selten Betriebsratsmitglieder Fälle von Entgeltungleichheit nicht auf, teils sogar im Einvernehmen mit den betroffenen Frauen, weil sie diese Spannungen innerhalb der Belegschaft nicht riskieren wollen.
Gibt es im Betrieb keinen Betriebsrat, fehlt es an einer Instanz, die bei der Korrektur der betrieblichen Entgeltsysteme und Entgeltpraxis die Interessen der Beschäftigten einbringen und durchsetzen kann. Das hat zur Folge, dass die/der Arbeitgeber/ in die diskriminierungsfreie Entgeltgestaltung wenigstens mit sachverständiger Begleitung entwickeln muss.

\section{Verfahren bei Tarifverträgen}

Wie gesagt, unterfallen auch Tarifverträge dem Entgeltgleichheitsgebot. Und nicht immer, oder besser gesagt, eher selten erfüllen sie es - wobei einige Gewerkschaften, zum Beispiel die IG Metall, bemerkenswerte Erfolge vorzeigen können. Die unmittelbaren Benachteiligungen wegen des Geschlechts sind zwar inzwischen verschwunden, die Arbeitsbewertung hingegen ist noch immer allzu häufig von Stereotypen geprägt, die weibliches Arbeitsvermögen und von Frauen besetzte Arbeitsplätze gering einschätzen. Tarifverträge bedürfen daher der Überprüfung und gegebenenfalls Korrektur. Das allerdings ist eine Herkulesaufgabe, denn hierzulande gelten zur Zeit über 50.000 Tarifverträge. Jährlich werden zwischen 6.000 und 7.000 von ihnen erneuert, Lohn- und Gehaltstarifverträge in der Regel alle ein bis zwei Jahre, Rahmen- und Manteltarifverträge, die die allgemeinen Arbeitsbedingungen und auch die Arbeitsbewertungsverfahren regeln, in größeren Abständen. ${ }^{8}$ Das geltende Recht verpflichtet die Tarifvertragsparteien, sich selbst um diskriminierungsfreie Regelungen zu bemühen; diskriminierende Regelungen sind nach der Rechtsprechung unwirksam. Das Gesetz sieht nunmehr vor, dass die ADS Tarifverträge, die Entgelte betreffen, einer kursorischen Prüfung im Hinblick auf Anhaltspunkte für Entgeltdiskriminierung unterzieht. Dies geschieht „auf Veranlassung“, so grundsätzlich bei Abschluss eines neuen Tarifvertrages oder auf Verlangen von Beschäftigten aus einem Betrieb ohne Betriebsrat. Die ADS soll damit entlastet werden von der Prüfung aller geltenden Tarifverträge - eine Lücke, die akzeptabel erscheint angesichts der Zahl der Tarifverträge und der Möglichkeit, über die betriebliche Prüfung auch an tarifliche Diskriminierungen heranzukommen. Zwar ist die Prüfung kursorisch, aber zunächst entsteht ein erheblicher Arbeitsaufwand für die ADS, der jedoch stark abnimmt nach der ersten Überprüfung - die Wiederholungsprüfung muss ja nur noch inzwischen eingetretene Veränderungen verarbeiten. Die Überprüfung jährlich abgeschlossener Entgelttarifverträge wird schon deshalb unkompliziert und schnell gehen, weil es sich ja hier nicht um Arbeitsbewertungsverfahren handelt.

Ergibt die kursorische Prüfung eines Tarifvertrages Anhaltspunkte für Entgeltdiskriminierung, sind die Tarifvertragspartei-

6 Vgl. dazu Kocher, Eva, „Die Anpassung diskriminierender Tarifverträge an das Recht“, in: djbZ 3/2010, S. 128-131; anders Raasch, Sibylle (2009, also noch vor der Entscheidung des EuGH), „,Gender Pay Gap mit neuen Instrumenten überwinden“, in: djbZ 3/2009, S. 126129, jeweils mit weiteren Nachweisen.

7 EuGH v. 8.9.2011, C-297/10 und C-298/10, Hennings und Mai; es ging dabei um Übergangsregelungen zur Abschaffung der Altersdiskriminierung im TVöD.

8 WSI Tarifarchiv <http://www.boeckler.de/wsi-tarifarchiv_3396. htm> (Zugriff: 29.6.2012). 
en zu einer detaillierten Prüfung verpflichtet. Bleiben sie während der ihnen eingeräumten Frist untätig, können diese Prüfung auch Antidiskriminierungsverbände vornehmen. Bestätigt die detaillierte Prüfung den Verdacht der Entgeltdiskriminierung, sind die zuständigen Tarifvertragsparteien aufgefordert, die diskriminierenden Regelungen durch diskriminierungsfreie zu ersetzen. Das dürfte nicht leicht sein, denn die abschließenden Tarifvertragsparteien haben gegensätzliche Interessen: die Gewerkschaften werden versuchen, das Entgeltniveau der bisher Benachteiligten auf das der Bevorzugten anzuheben, die Arbeitgeberseite wird die damit verbundene Personalkostensteigerungen vermeiden wollen. Dies voraussehend sieht das Gesetz vor, dass auf Antrag einer zuständigen Tarifvertragspartei oder des Antidiskriminierungsverbandes, der die detaillierte Prüfung durchgeführt hat, das Arbeitsgericht eine vorläufige Regelung treffen kann, wonach das Entgelt nach oben anzupassen ist, bis ein diskriminierungsfreier Tarifvertrag abgeschlossen worden ist. Diese Regelung wird den Einigungsdruck erhöhen, denn nur die Tarifvertragsparteien selbst dürfen Stufenpläne und Übergangsfristen festlegen, wenn es die wirtschaftliche Lage von betroffenen Betrieben im Tarifbereich gebietet.

\section{Sanktionen}

Das Gesetz arbeitet sehr zurückhaltend mit Sanktionen; sie werden nur bei Untätigkeit der Arbeitgeber/innen ausgelöst. Die gesetzlichen Verpflichtungen gegenüber der ADS können durch eigenes staatliches Handeln wie der Verhängung von Ordnungsgeldern durchgesetzt werden. Aber der ADS schuldet die/der Arbeitgeber/in ja nur Berichte und Daten, die zur Verfügung zu stellen sind. Die Auswirkungen der Zurückhaltung des Gesetzentwurfes gegenüber flächendeckenden staatlichen Eingriffen werden hier deutlich. Denn es würde gewiss öfter vorkommen, dass die/der Arbeitgeber/in zwar Berichte und Daten liefert, die Verdachtsmomente für eine Diskriminierung aber schlicht ignoriert und die diskriminierende betriebliche Entgeltpraxis einfach fortführt. Hier käme es dann ganz auf die eigenbestimmte Aktivität derjenigen an, die das Gesetz mit Einwirkungsrechten ausstattet, ohne aber Einwirkungspflichten zu begründen. Blieben auch sie untätig, wenden sie sich nicht an das Arbeitsgericht, um ein Tätigwerden zu erzwingen, geschieht gar nichts. Sicherlich würden Betriebsräte im entsprechenden Fall nicht ähnlich ignorant reagieren, wie es seitens der Arbeitgeber/innen zu erwarten ist. Die Betriebsräte würden wohl auch durch die nunmehr besser informierten Beschäftigen in Zugzwang gebracht werden. Mehr noch wäre aber eine Änderung des Verhaltens durch Aktivitäten der Antidiskriminierungsverbände zu erhoffen. Diese würden sich aus den Listen der ADS über Betriebe, bei denen Entgeltdiskriminierung festgestellt worden ist, gewiss zunächst jene heraussuchen, die viele Beschäftigte haben und bei denen das Verfahren auch auf öffentliche Aufmerksamkeit stoßen wird. Das würde andere Unternehmen dazu bringen, doch von sich aus tätig zu werden, um diese negative Aufmerksamkeit zu vermeiden. So könnte schließlich eine für das Anliegen Entgeltgleichheit hilfreiche Wettbewerbssituation entstehen. Denn Betriebsräte und
Gewerkschaften würden sich ungern von Antidiskriminierungsverbänden in den Schatten stellen lassen. Voraussetzung ist allerdings, dass die Annahme der SPD-Bundestagsfraktion zutrifft, es würden ausreichend Antidiskriminierungsverbände gebildet werden, die diese segensreiche Tätigkeit aufnehmen.

\section{Ausblick}

Der Gesetzentwurf der SPD wird nicht Gesetz werden, jedenfalls nicht in der laufenden Legislaturperiode. Das macht das Protokoll der ersten Lesung des Gesetzentwurfes am 14. Juni $2012^{9}$ deutlich; die Parteien der Regierungskoalition haben ihn rundheraus abgelehnt, die Linke vermisst ein Verbandsklagerecht, die Grünen unterstützen den Entwurf und kündigen Verbesserungsvorschläge an, was ja nur zu begrüßen ist. Mit dem Ende der Legislaturperiode wird dieser Gesetzentwurf im Prinzip Makulatur. Die Tatsache, dass die SPDBundestagsfraktion nicht nur allgemeine Erklärungen über eine Bekämpfung der Entgeltungleichheit abgegeben, sondern einen ausformulierten und konkreten Gesetzentwurf vorgelegt hat, lässt hoffen. Hoffen darauf, dass sie diese Entschlossenheit auch dann zeigt, wenn sie wieder in Regierungsverantwortung steht. Frauen haben da ja in der jüngeren Vergangenheit bei einem Gleichstellungsgesetz für die Privatwirtschaft durchaus böse Erfahrungen gemacht, die einer Wiederholung nicht bedürfen.

Zwar nicht überraschend, aber wegen ihrer Schlichtheit doch entmutigend waren die Begründungen für die Ablehnung: Das Problem der Entgeltungleichheit sei einfach zu lösen durch eine bessere Vereinbarkeit von Beruf und Familie, durch unmittelbar zu erwartendes einsichtsvolles Handeln der Unternehmen und vor allem durch Verhaltensänderungen bei den Frauen selbst. Der Gesetzentwurf wird als Bürokratiemonster bezeichnet, der durch übermäßige Kosten die Wettbewerbsfähigkeit der deutschen Wirtschaft vernichten würde. Besonders beeindruckend ist immer wieder der pathetische Kampf der Koalitionsparteien für die Gewerkschaften und die Tarifautonomie, wenn es darum geht, unter Berufung auf sie sozialpolitisch notwendige Gesetze zu verhindern. Die Tarifvertragsparteien würden es schon richten, wird behauptet, ja, mehr noch, sie hätten es schon getan. ${ }^{10}$ Über die keineswegs flächendeckende und abnehmende Verbreitung von Tarifverträgen ${ }^{11}$ wird dabei großzügig hinweggesehen. Übrigens ist der Gesetzentwurf im Vorfeld mit den Gewerkschaften beraten worden; bei einigen verbliebenen Kritikpunkten akzeptieren sie dessen Ansatz.

Zur Unterstützung bedarf ein gesetzliches Vorhaben zur Durchsetzung der Entgeltgleichheit einer öffentlichen Diskussion, an der sich hoffentlich der djb, wie schon in der Vergangenheit, intensiv beteiligen wird.

9 〈http://dip21.bundestag.de/dip21/btp/17/17184.pdf> (Zugriff: 29.6.2012).

10 Die letzten Urteile des BAG zu Geschlechtsdiskriminierungen in Tarifverträgen sind so lange nicht her, nämlich aus 2009 und 2006 (NZA 2009, S. 785; 2006, S. 1411).

11 S. dazu WSI-Tarifarchiv <http://www.boeckler.de/ wsi-tarifarchiv_4828.htm> (Zugriff: 29.6.2012). 